\title{
Self-initiated behavioural change and disease resurgence on activity-driven networks
}

\author{
Nicolò Gozzi, ${ }^{1}$ Martina Scudeler, ${ }^{2}$ Daniela Paolotti, ${ }^{3}$ Andrea Baronchelli, ${ }^{4,5}$ and Nicola Perra ${ }^{1, *}$ \\ ${ }^{1}$ Networks and Urban Systems Centre, University of Greenwich, London, UK \\ ${ }^{2}$ University of Turin, Turin, Italy \\ ${ }^{3}$ ISI Foundation, Turin, Italy \\ ${ }^{4}$ City, University of London, London, UK \\ ${ }^{5}$ The Alan Turing Institute, London, UK
}

(Dated: June 24, 2021)

\begin{abstract}
We consider a population that experienced a first wave of infections, interrupted by strong, top-down, governmental restrictions and did not develop a significant immunity to prevent a second wave (i.e., resurgence). As restrictions are lifted, individuals adapt their social behaviour to minimize the risk of infection. We explore two scenarios. In the first, individuals reduce their overall social activity towards the rest of the population. In the second scenario, they maintain a normal social activity within a small community of peers (i.e., social bubble) while reducing social interactions with the rest of the population. In both cases, we investigate possible correlations between social activity and behaviour change, reflecting for example the social dimension of certain occupations. We model these scenarios considering a Susceptible-Infected-Recovered epidemic model unfolding on activity-driven networks. Extensive analytical and numerical results show that i) a minority of very active individuals not changing behaviour may nullify the efforts of the large majority of the population, and ii) imperfect social bubbles of normal social activity may be less effective than an overall reduction of social interactions.
\end{abstract}

\section{INTRODUCTION}

The spreading of infectious diseases and human behaviour are fundamentally intertwined [1-6]. On one side, the unfolding of epidemics might induce people to modify social contacts, habits, and mobility. On the other, such changes might drastically affect the course of outbreaks.

Behavioural change is a blanket term used to describe a wide range of (re)actions. More in detail, these can be classified into two main categories. The first consists of bottom-up, self-initiated changes implemented by individuals according to their perceived risk and susceptibility as well as to the perceived barriers and benefits linked to each action [7-10]. These individual decisions vary from social distancing and increased hygiene to the adoption of healthy diets and the use of personal protective equipment such as face masks [1, 2]. The second category instead, describes top-down, governmental, interventions aimed at interrupting chains of infection banning (or limiting) large gatherings, mobility within and across countries, as well as strict lockdowns and cordon sanitarie $[1,2,6,11]$.

The literature on the subject provides a wealth of theoretical models developed to capture behavioural change and characterise their effects on diseases [1,2]. These studies differ according to the level of analysis, from single homogeneously mixed populations to individual based contact networks, and according to the mechanisms adopted to model changes in behaviours. Several works tackle the problem by considering variations in individuals' features or in diseases' parameters [12-17], while others focus on changes in connectivity patterns [4, 18-21]. Across the board, such variations are linked to i) disease prevalence and/or ii) individuals' beliefs and (mis)information circulating in the system. The first

*n.perra@greenwich.ac.uk approach, typically, does not affect the threshold properties of the spreading. In fact, in this case, behavioural change starts to be implemented only after the initial growth of the infected population. Nonetheless, prevalence-induced behavioural change can drastically reduce the final disease burden. The second approach, instead, can also affect threshold properties and thus modify the conditions necessary for a macroscopic outbreak even in the case of simple, homogeneously mixed populations $[12,22]$.

Unfortunately, the COVID-19 pandemic highlights the importance of behavioural changes across the various phases of the emergency [6]. For example, after the first wave, many countries gradually lifted the top-down measures implemented to curb the spreading of the SARS-CoV-2 virus. Such interventions have been largely induced by the local spreading and in particular by the burden to the healthcare systems. When they were relaxed, self-initiated behavioural change (nudged by new regulations) became fundamental. Indeed, evidence from serological studies and modelling efforts indicated that the immunity resulting from the first wave was very far from the one required for herd immunity [23-25]. In this context, we present a theoretical framework aimed at investigating the effects of behavioural changes on disease resurgence on time-varying contact networks [26-28]. In particular, we consider the following, unfortunately realistic, scenario. We imagine a population that experienced a first wave of infections which due to strict, top-down measures, was interrupted early. We imagine that interventions are lifted and that people reduce their social interactions (respect to the usual baseline) either because mindful of the risk of propagating the virus (if infected) or because concerned about the risk of infection (if not infected). In doing so, we explore the effects on disease resurgence if such changes are implemented only by a fraction of the population selected i) at random and ii) according to the propensity individuals have to establish social interactions. We adopt activity-driven 
networks, a class of time-varying networks, to model the temporal interactions between individuals [29-34]. Although they are a simple approximation of real contact networks, they capture an important property of human interactions: the heterogeneity of human activity. In fact, evidence from a wide range of real datasets capturing human interactions in various contexts suggests that the propensity per unit time of people to establish social connections (i.e., the activity), is highly heterogeneous [29-31, 35, 36]. Here, we first consider the basic formulation of the model in which, at each time step, active nodes create random connections with others [29]. In these settings, we derive the analytical expression of the epidemic threshold of a Susceptible-Infected-Recovered model [37] unfolding on top of the temporal networks as a function of the parameters and mechanisms defining the behavioural changes. In particular, we consider the case of partial adoption of such changes and find a closed-form expression for the basic reproductive number $R_{0}$. If adoption is assigned in increasing order of activity an almost perfect level of conformity is required to avoid disease resurgence. This highlights that the lack of adoption of a small number of highly socially active individuals may jeopardize large collective efforts. We then consider a more realistic variation of activity-driven networks able to capture the mesoscopic organisation of real sociograms in tightly connected groups (i.e., communities) $[34,38]$. In these settings, we rely on numerical simulations to characterise the effects of behavioural changes. In doing so, we consider two different types of adaptive behaviours. The first is a reduction of activity. The second instead is inspired by the concept of social bubbles: nodes keep their social propensity but they direct it towards a small social group. We model this scenario by increasing the probability of interactions within communities. Results show that the presence of communities increases the threshold making it more difficult for a disease to spread. Furthermore, behavioural changes aimed at reducing activity have a much stronger effect on the spreading with respect to those aimed at increasing the cohesiveness of small social groups.

The paper is organised as follows. In Section II we introduce activity-driven networks. In Section III we describe the spreading of infectious diseases on this class of time-varying networks. In doing so, we first describe the various mechanisms of behavioural changes induced in the population and then characterize their effects on the spreading of the disease. In Section IV we present our conclusions.

\section{ACTIVITY-DRIVEN NETWORKS}

In activity-driven networks, the temporal interactions between $N$ nodes are captured by two steps. The first is the node activation defining the subset of nodes that, at each time step, are active and willing to establish social interactions. The second is the partner selection defining with whom each active node will connect to. Nodes' activation is modelled assigning to each node an activity $a$. This quantity, extracted from a distribution $F(a)$, describes the rate at which each node is active per unit time. Hence, the inter-event time between two activations of each node is distributed as a Poissonian with average 1/a. Extensions of the activity-driven framework to nonPoissonian activation patterns have been proposed [32, 39], but for simplicity are not considered here. As mentioned above, observations in several real networks suggest that such distribution is heterogeneous [29-31, 35, 36]. For simplicity, in the following we assume $F(a) \sim a^{-\alpha}$ with $\epsilon \leq a \leq 1$ to avoid divergences. Several mechanisms have been proposed for the second step [29-34, 40]. Here, we consider two: random and community-based partner selection.

\section{A. Random partner selection}

In the basic formulation of the model, each active node creates $m$ random connections with others [29]. Connections are done without recollection of past interactions. Thus, the process is Markovian. In these simple settings, the network's temporal dynamics can be summarised as follows:

- at each time $t$, the network $G_{t}$ is initially disconnected;

- each node is active with probability $a \Delta t$ creating $m$ random connections;

- each connection is deleted, time incremented to $t+\Delta t$, and the process restarts from the first point.

In other words, each connection lasts for a $\Delta t$ duration (without loss of generality here we set $\Delta t=1$ ) and it is created randomly by active nodes. Thus, at each time step, the network $G_{t}$ is mostly made up of disconnected stars centered around active nodes. It can be easily shown that the distribution of the number of connections of each node (i.e., the degree) in the aggregated network obtained integrating links over several time-steps follows the distribution of the activity [29, 41]. Hence, heterogeneous activity patterns induce the formation of hubs which are highly active nodes engaging over and over in social interactions. However, since links are created at random, the distribution of links' weights in the time-integrated network is homogeneous and thus very far from observations in real networks [42-44]. In summary, this version of the model captures some important features of real systems and it allows for analytical analyses of dynamical processes unfolding on its structure at comparable time-scales [21, 29, 45-50], but at the same time it is a rough approximation of real social networks.

\section{B. Community-based partner selection}

This second approach considers a much more realistic partner selection mechanism. In fact, social networks are organised in tightly connected groups (i.e., communities), which emerge and evolve in time [38]. As a result, the vast majority of connections takes place within such circles of friends rather than across them [42, 43]. To capture this fundamental aspect of social interactions, each node is assigned to a particular community $c$. The size $s$ of each community is extracted 
from a distribution $G(s)$ [34]. In these settings, the dynamics of the network follows these steps:

- at each time $t$, the network $G_{t}$ is initially disconnected;

- each node is active with probability $a \Delta t$ creating $m$ connections;

- with probability $\eta$ each connection is done selecting at random one of the nodes in the same community and with probability $1-\eta$ selecting at random in any other community;

- each connection is deleted, time incremented to $t+\Delta t$ and the process restarts from the first point.

As done above, without loss of generality we set $\Delta t=1$. The parameter $\eta$ regulates the modularity of the emerging network. For $\eta=0$ (and in case of community sizes $s \ll N$ ) the network unfolds very similarly to the first model. Instead for $\eta=1$ the network will be formed by completely disconnected communities. The link creation dynamics are still Markovian since no memory of past interactions is used to inform the partner selection process.

\section{BEHAVIOURAL CHANGES INDUCED BY DISEASE SPREADING ON ACTIVITY-DRIVEN NETWORKS}

We study the spreading of an infectious disease unfolding at a comparable time-scale with respect to the evolution of connections in the contact network. We consider the prototypical Susceptible-Infected-Recovered epidemic model [37, 51]. Thus, each node can be found in one of three compartments: healthy and susceptible individuals are in the compartment $S$, infectious in $I$, and recovered in $R$. The disease propagates via the connections between susceptible and infectious nodes with a probability of infection, per contact, $\lambda$. Infected nodes recover spontaneously with probability $\mu$. Hence, we consider the epidemic dynamics as Markovian. Modeling frameworks able to account for non-Markovian features have been proposed [52-54]. Such features have been shown to affect the spreading. However, in the case of SIS dynamics, recent results point to the equivalence between the two given an adequate rescaling of the parameters [55]. In the case of an outbreak without residual immunity (i.e., $R(t=0)=0$ ), the epidemic threshold in a memoryless activity-driven network can be obtained, using a mean-field approach, studying the evolution of the number of infected nodes with activity $a$. In particular, this threshold reads [29]:

$$
\frac{\lambda}{\mu}>\frac{1}{m} \frac{1}{\langle a\rangle+\sqrt{\left\langle a^{2}\right\rangle}}
$$

which implies that the disease will be able to spread only if $R_{0}=m \frac{\lambda}{\mu}\left(\langle a\rangle+\sqrt{\left\langle a^{2}\right\rangle}\right)>1$. In this last expression, we introduced the basic reproductive number $R_{0}$ as the number of secondary infections generated by an index case in a fully susceptible population [37]. Interestingly, the threshold of a SIR (for a SIS the threshold is the same) model is driven by the first and second moment of the activity distribution rather than the time-aggregated properties of the graph.

In the case of activity-driven networks with communities, we do not have a closed expression for the threshold. However, it is interesting to notice how the presence of communities affects SIR and SIS models very differently [34]. In fact, while in the case of permanent immunity (SIR) the repetition of contacts within communities hampers the spreading, it helps the diffusion of diseases able to reach an endemic state (SIS). In other words, modularity pushes the threshold of SIR models to higher values while facilitates the spreading of SIS models, pushing the threshold to lower values. Similar results have been obtained in case of non-Markovian partners' selection processes induced by memory of past interactions [56, 57]. These allow for the emergence of weak and strong ties, impose the repetition of few connections that break the symmetry between SIR and SIS epidemic models.

As mentioned in the introduction, in this work we investigate the following scenario. A highly infectious disease spreads in the network, but its course is halted by strict topdown interventions. In this first wave, the large majority of individuals has not been affected, thus the system is far from herd immunity. As the measures are lifted, individuals, maybe nudged by laws and regulations, implement behavioural changes with the aim of protecting themselves and the others and thus avoid - or mitigate - a second wave.

Rizzo et al [21] have studied the implementation of behavioural changes reducing the activity of susceptible by a factor $\gamma$ and the activity of infected by a factor $\psi$. Here, we extend this approach by studying the effect of adoption rates. We consider that such changes in behaviour are implemented only by a fraction of nodes selected either at random or as a function of the activity. In fact, as suggested by the healthbelief model [7, 8], the adoption of behavioural changes is influenced by the barriers associated with their implementation. Very active people, maybe due to their occupation, might be more penalized by reducing their activity and thus less likely to adhere. As clearly shown during the COVID-19 pandemic however, the adoption of non-pharmaceutical interventions is a complex issue linked to age, gender, education, socio-economics factors, political view, religion and other beliefs [6].

As a second step, we further extend the literature exploring the interplay between behavioural changes and the modularity of the network. This corresponds to the fact that individuals might keep the same activity but cut connections with people outside their close-knit social circles. Also in this case, we study the role of adoption considering only a fraction of nodes engaging in any form of behavioural change.

\section{A. Non-perfect adoption}

Following the order described above, we first consider a scenario in which, as a way to reduce the risk of infection, susceptible individuals reduce their activity of a factor $\gamma$ and infected by a factor $\psi$. However, we assume that only a fraction of the population is willing - or able - to reduce the ac- 
tivity. For this reason, we first consider that the propensity to implement behavioural changes is independent of node's features and only function of its status. Thus, across all activity classes, only a random fraction $p$ of susceptible and a fraction $w$ of infected will reduce the activity. Assuming that nodes of the same activity are statistically equivalent, we can write the evolution of the number of infected node of activity class $a$ as:

$$
\begin{aligned}
d_{t} I_{a} & =-\mu I_{a}+m \lambda\left(N_{a}-I_{a}-R_{a}\right) a \gamma_{p} \int d a^{\prime} \frac{I_{a^{\prime}}}{N}+ \\
& +m \lambda\left(N_{a}-I_{a}-R_{a}\right) \psi_{w} \int d a^{\prime} \frac{I_{a^{\prime}} a^{\prime}}{N}
\end{aligned}
$$

where $N_{a}=S_{a}+I_{a}+R_{a}$ holds for all activity classes, $\gamma_{p}=$ $1-p(1-\gamma)$ and $\psi_{w}=1-w(1-\psi)$. The first term in the right hand side describes the recovery process. The second captures susceptible nodes that become active and select as partner an infected individual in any other activity class. The third term, instead, describes susceptible nodes that are selected by active and infected nodes in any activity class. In the early stages of the possible second wave, we assume $I_{a} \ll S_{a}$ and $R_{a} \ll S_{a}$ thus $N_{a} \sim S_{a}$. In other words, the first wave was stopped well before the disease was able to affect a large fraction of the population. In these settings we drop all the second order terms obtaining:

$$
d_{t} I_{a}=-\mu I_{a}+m \lambda N_{a} a \gamma_{p} \int d a^{\prime} \frac{I_{a^{\prime}}}{N}+m \lambda N_{a} \psi_{w} \int d a^{\prime} \frac{I_{a^{\prime}} a^{\prime}}{N} .
$$

By integrating both sides over all activity classes we have:

$$
d_{t} I=-\mu I+m \lambda \gamma_{p}\langle a\rangle I+m \lambda \psi_{w} \Theta
$$

where $\Theta=\int d a I_{a} a$ and $\left\langle a^{n}\right\rangle=\int d a F(a) a^{n}$. To characterise the evolution of $I(t)$ we then need to derive an equation for $\Theta$. In particular, multiplying both sides of Eq. 3 by $a$ and integrating across all activities:

$$
d_{t} \Theta=-\mu \Theta+m \lambda \gamma_{p}\left\langle a^{2}\right\rangle I+m \lambda \psi_{w}\langle a\rangle \Theta .
$$

The epidemic threshold can be obtained studying the stability of the system of differential equations defined by Eq. 4 and Eq. 5. Indeed, the disease will be able to spread only if the largest eigenvalue of the Jacobian matrix $J$ of the system is larger than zero. Here, $J$ can be written as:

$$
J=\left(\begin{array}{cc}
-\mu+m \lambda \gamma_{p}\langle a\rangle & m \lambda \psi_{w} \\
m \lambda \gamma_{p}\left\langle a^{2}\right\rangle & -\mu+m \lambda \psi_{w}\langle a\rangle
\end{array}\right)
$$

And the threshold, is:

$$
\frac{\lambda}{\mu}>\frac{2}{m} \frac{1}{\langle a\rangle\left(\gamma_{p}+\psi_{w}\right)+\sqrt{\left(\gamma_{p}-\psi_{w}\right)^{2}\langle a\rangle^{2}+4 \gamma_{p} \psi_{w}\left\langle a^{2}\right\rangle}}
$$

This condition implies $R_{0}=(\lambda / \mu) \mathcal{T}^{-1}>1$, where $\mathcal{T}$ is the right hand side Eq. 6 . Notably, when $\gamma=\psi=1$, it reduces to the threshold of activity-driven networks without behavioural changes. However, it is interesting to notice how these two reductions of activity rates do not imply a simple rescaling of the threshold. Indeed, they introduce non-linear terms in the expression. Furthermore, for $p=w=1$, the threshold reduces to the expression obtained by Rizzo et al [21] describing the case of perfect adoption. The threshold is symmetric in $\gamma_{p}$ and $\psi_{w}$ meaning that the effective reduction in activity of susceptible $\left(\gamma_{p}\right)$ and of infected $\left(\psi_{w}\right)$ can be switched without implying any change in the threshold. It is important to notice, however, that in the early stages of the outbreak the number of susceptible is much larger than the number of infected. Thus, since reductions of activity come with high social costs, combinations of parameters with lower values of $\psi_{w}$ rather than $\gamma_{p}$ are desirable.

We can see these effects in Fig. 1-a where we show $R_{0}$ (calculated from Eq. 6) as a function of $\gamma$ and $\psi$. As expected, in the case of random adoption the epidemic threshold is shifted towards lower values of $\gamma$ and $\psi$ with respect to perfect adoption (dashed line). This implies that larger reductions of activity are required in order to stop the disease from spreading. In Fig. 1-b, instead, we show $R_{0}$ as a function of $p$ and $w$ for fixed values of $\gamma$ and $\psi$. We observe that $p$ and $w$ interpolate between two opposite regimes. By reducing their values, the adoption of behavioural changes becomes increasingly less significant. Consequently, $R_{0}$ assumes values closer to those described in Eq. 1 which captures a system without behavioural changes. Conversely, by increasing the values of $p$ and $w$, the values of $R_{0}$ become progressively closer to a system with $100 \%$ adoption of behavioural changes.

We test the analytical solution derived in Eq. 6 by means of numerical simulations. In particular, we consider a case in which infected individuals reduce their activity more (due to their illness status, for example) with respect to the susceptible by setting $\gamma=0.8$, and $\psi=0.1$. In Fig. 2-a, we plot $r_{\infty}=R_{\infty} / N$ as a function of $\lambda$, for different values of $p$ and $w$. The epidemic size grows with the infectiousness of the pathogen and it decreases when a larger fraction of individuals implement behavioural changes. As expected from the theory, the final epidemic size obtained with $80 \%$ of susceptible and infected implementing behavioural changes is very similar to the one obtained with only $10 \%$ of susceptible and $80 \%$ of infected engaging in such changes. Taking into account that at the beginning of the possible second wave the number of infected is much smaller than the number of susceptible, and given the high socio-economic cost of isolating individuals, a setting in which infected reduce their social interactions more is clearly desirable. In Fig. 2-b, instead, we represent, for different combinations of parameters, the relative variance $\sigma_{r_{\infty}}$ of the final epidemic size as a function of $\lambda$ [13]. This is defined as $\sigma_{r_{\infty}}=\sqrt{\left\langle r_{\infty}^{2}\right\rangle-\left\langle r_{\infty}\right\rangle^{2}} /\left\langle r_{\infty}\right\rangle$. Because of the critical behaviour of the epidemic process we are considering, the maximum $\sigma_{r_{\infty}}$ is reached at the threshold. As expected, we observe that, for the different combinations of parameters considered, the normalized relative variance peaks around the theoretical threshold values, providing a numerical validation of the analytical expression found previously. 


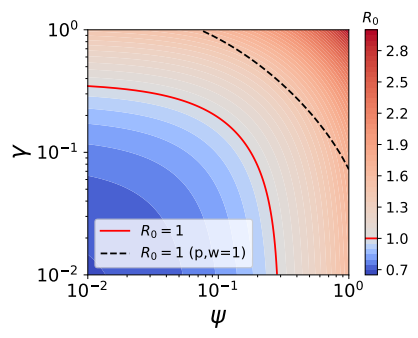

(a)

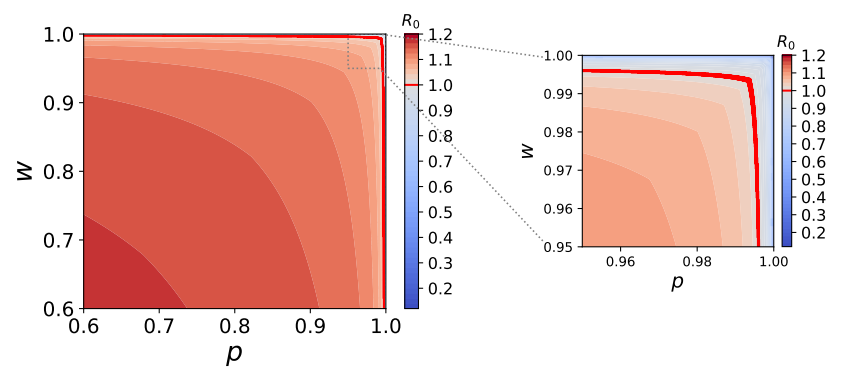

(c)

FIG. 1: We show the analytical value of the basic reproductive number $R_{0}$ in different scenarios. a) $R_{0}$ as a function of $\gamma$ and $\psi$ as obtained in Eq. 6 in the case of non-perfect adoption independent of nodes' activity. We set $p=0.75$ and $w=0.8$. b) $R_{0}$ as a function of $p$ and $w$ as obtained in Eq. 6 in the case of non-perfect adoption independent of nodes' activity. We set $\gamma=0.1$ and $\psi=0.1$. c) $R_{0}$ as a function of $p$ and $w$ as obtained in Eq. 20 in the case of adoption dependent from nodes' activity. We set $\gamma=0.1$ and $\psi=0.1$. The panel on the right highlights a small region of the phase space. In all figures we set $\epsilon=10^{-3}, m=2, \alpha=2.1, \mu=10^{-2}$, and we indicate with a solid red line the threshold $R_{0}=1$. The parameters are set so that when $\gamma=1, \psi=1$ (i.e., without behavioural changes) $R_{0}=2$ in panel a), while $R_{0}=1.2$ in panels b) and c).

\section{B. Non-perfect adoption depending on activity}

We now consider the case in which the propensity of implementing behavioural changes is linked to the activity. We can imagine that, due to the higher barriers and costs associated with the change in behaviour, nodes with larger activity are less prone to such changes. A natural example are individuals that due to the nature of their job cannot easily reduce the number of their interactions. For simplicity, we assume that only susceptible nodes with activity lower or equal to $a_{p}$ and infected nodes with activity lower or equal than $a_{w}$ implement behavioural changes. In other words, nodes with activity larger than a given threshold will not change their behaviour. As mentioned above, adoption of behavioural changes is a complex issue shaped by many factors. Thus, our modeling approach here is just a simplified approximation amenable to highlight the possible interplay between activity and adoption rates. In these settings, the maximum fraction $x$ of individuals changing behaviour is linked to the activity cut-off value $a_{x}$ as follows:

$$
x=\int_{\epsilon}^{a_{x}} F(a) d a .
$$

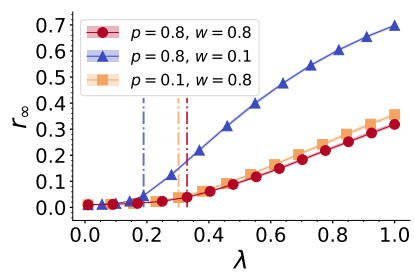

(a)

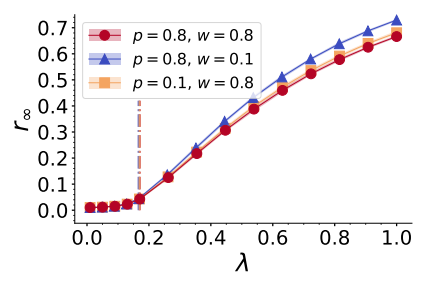

(c)

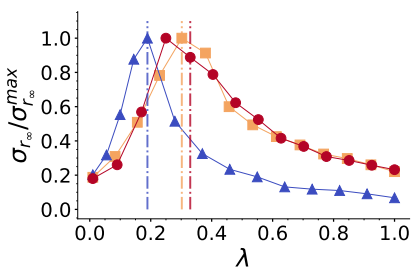

(b)

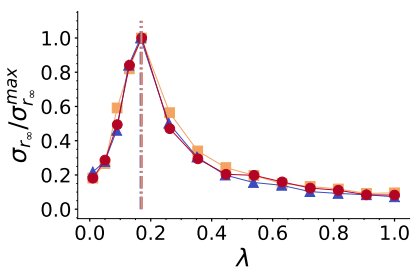

(d)
FIG. 2: In panel a) we display the final epidemic size $\left(r_{\infty}=R_{\infty} / N\right.$ ) with $95 \%$ confidence intervals and in panel $\mathrm{b}$ ) the normalized relative variance $\sigma_{r_{\infty}} / \sigma_{r_{\infty}}^{\max }$ for different values of $\lambda$ in the case of non-perfect random adoption. Vertical dashed lines indicates the analytical threshold derived from Eq. 6 for the different values of $p$ and $w$ considered. In panel c) and d) we repeat the analysis in the case of non-perfect adoption dependent from nodes' activity for different values of $p$ and $w$. In all figures, we represent median and $95 \%$ confidence intervals obtained from $10^{2}$ stochastic simulations for each point with the following parameters $\mu=10^{-2}$, $N=10^{6}, m=2, \epsilon=10^{-3}, \alpha=2.1, i_{0}=I_{0} / N=0.01$ (initial fraction of infected seeds), $\gamma=0.8, \psi=0.1$.

In general, since the health status of each node affects the type of behavioural change, the fraction of, say, infected nodes adopting behavioural change is smaller that the respective $x$. The equation regulating the change of number of infected in a specific activity class $a$ at early stages of the spreading can be written:

$$
\begin{aligned}
d_{t} I_{a} & =-\mu I_{a}+m \lambda N_{a} a\left[1-\theta\left[a_{p}-a\right](1-\gamma)\right] \int d a^{\prime} \frac{I_{a^{\prime}}}{N}+ \\
& +m \lambda N_{a} \int d a^{\prime}\left[1-\theta\left[a_{w}-a^{\prime}\right](1-\psi)\right] \frac{I_{a^{\prime}} a^{\prime}}{N},
\end{aligned}
$$

where $\theta[x-y]$ is a heaviside step function equal to one for $x \geq y$ and zero otherwise. Integrating over all activities and introducing $\Xi=\int d a^{\prime}\left[1-\theta\left[a_{w}-a^{\prime}\right](1-\psi)\right] I_{a^{\prime}} a^{\prime}$ we get:

$$
d_{t} I=-\mu I+m \lambda\left[\langle a\rangle+\langle a\rangle_{p}(1-\gamma)\right] I+m \lambda \Xi,
$$

where we defined $\left\langle a^{n}\right\rangle_{p}=\int_{\epsilon}^{a_{p}} F(a) a^{n} d a$ as the $\mathrm{n}^{t h}$ moments of the group of nodes adopting behavioural changes. In order to understand the early dynamics we need to get an equation for $\Xi$. To this end, we can multiply both terms of Eq. 8 for $\left[1-\theta\left[a_{w}-a\right](1-\psi)\right] a$ and integrating across all activities we obtain:

$$
\begin{aligned}
d_{t} \Xi & =-\mu \Xi+m \lambda \mathcal{F}\left[\left\langle a^{2}\right\rangle, \gamma, \psi, a_{p}, a_{w}\right] I+ \\
& +m \lambda\left[\langle a\rangle-\langle a\rangle_{w}(1-\psi)\right] \Xi,
\end{aligned}
$$


where we defined:

$$
\begin{aligned}
\mathcal{F}\left[\left\langle a^{2}\right\rangle, \gamma, \psi, a_{p}, a_{w}\right] & =\left\langle a^{2}\right\rangle-\left\langle a^{2}\right\rangle_{p}(1-\gamma)-\left\langle a^{2}\right\rangle_{w}(1-\psi) \\
& +\left\langle a^{2}\right\rangle_{\text {min }_{a_{p}, a_{w}}}(1-\gamma)(1-\psi)
\end{aligned}
$$

which is a modulation of the second moment of the activity distribution. From this stand point we can write the Jacobian matrix of the system of differential equations. To simplify further the notation, we define:

$$
\begin{aligned}
& \alpha_{p}=\langle a\rangle-\langle a\rangle_{p}(1-\gamma) \\
& \alpha_{w}=\langle a\rangle-\langle a\rangle_{w}(1-\psi)
\end{aligned}
$$

Therefore, the threshold behaviour is encoded in the system of differential equations:

$$
\begin{aligned}
& d_{t} I=\left(-\mu+\lambda m \alpha_{p}\right) I+\lambda m \Xi \\
& d_{t} \Xi=+\lambda m \mathcal{F} I+\left(-\mu+\lambda m \alpha_{w}\right) \Xi
\end{aligned}
$$

The disease will be able to grow only if the largest eigenvalue of the Jacobian matrix of this system is larger than zero. The Jacobian matrix can be written as:

$$
J=\left(\begin{array}{cc}
-\mu+\lambda m \alpha_{p} & \lambda m \\
\lambda m \mathcal{F} & -\mu+\lambda m \alpha_{w}
\end{array}\right)
$$

The eigenvalues $k_{1,2}$ can be found solving the characteristic equation:

$$
\begin{aligned}
& k^{2}+k\left(2 \mu-\lambda m\left(\alpha_{p}+\alpha_{w}\right)\right)+\lambda^{2} m^{2}\left(\alpha_{p} \alpha_{w}-\mathcal{F}\right) \\
& -\mu \lambda m\left(\alpha_{p}+\alpha_{w}\right)+\mu^{2}=0
\end{aligned}
$$

leading to:

$k_{1,2}=\frac{1}{2}\left[-2 \mu+\lambda m\left(\alpha_{p}+\alpha_{w}\right) \pm \lambda m \sqrt{\left(\alpha_{p}-\alpha_{w}\right)^{2}+4 \mathcal{F}}\right]$

The threshold condition can be written as:

$$
\frac{\lambda}{\mu}>\frac{2}{m\left[\left(\alpha_{p}+\alpha_{w}\right)+\sqrt{\left(\alpha_{p}-\alpha_{w}\right)^{2}+4 \mathcal{F}}\right]}
$$

Substituting the values of $\alpha_{p}, \alpha_{w}, \mathcal{F}$ with those from equation $11,12,13$, we obtain the threshold as a function of the activity moments:

$$
\frac{\lambda}{\mu}>\frac{2}{m\left[2\langle a\rangle-(1-\gamma)\langle a\rangle_{p}-(1-\psi)\langle a\rangle_{w}+\sqrt{\Delta}\right]}
$$

where

$$
\begin{aligned}
\Delta & =4\left\langle a^{2}\right\rangle+(1-\gamma)\left[\langle a\rangle_{p}^{2}(1-\gamma)-4\left\langle a^{2}\right\rangle_{p}\right] \\
& +(1-\psi)\left[\langle a\rangle_{w}^{2}(1-\psi)-4\left\langle a^{2}\right\rangle_{w}\right] \\
& +2(1-\gamma)(1-\psi)\left[2\left\langle a^{2}\right\rangle_{\min \left(a_{p}, a_{w}\right)}-\langle a\rangle_{w}\langle a\rangle_{p}\right](21)
\end{aligned}
$$

We can verify that, if $a_{p}=a_{w}=1$, we find again the result of Eq. 6. In Fig. 1-c we compare the effects of different values of $p$ and $w$ on $R_{0}$ in the case of activity-based adoption using Eq. 7 to compute the correspondent values of $a_{p}$ and $a_{w}$. Very differently from the case of a random adoption (Fig. 1-b), almost perfect conformity to the behavioural measures is needed to halt the spreading and push $R_{0}$ below 1 . Indeed, even if the majority of individuals reduces their activity, the interactions of the most active nodes are sufficient to sustain the spreading. This is in line with the study of immunization strategies in activity-driven networks [46]. In fact, the strategic immunization of few central, most active, nodes has been shown sufficient to halt outbreaks. This implies that these nodes are indeed key to the unfolding of the virus. In Fig. 2-c-d we test the analytical solution by means of numerical simulations. We plot $r_{\infty}$ and $\sigma_{r_{\infty}} / \sigma_{r_{\infty}}^{\max }$ as functions of $\lambda$, for different values of $p$ and $w$. Interestingly, we observe that large differences in $p$ and $w$ result in very similar attack rate profiles and thresholds. This confirms how the spreading is controlled by the most active nodes that are not compliant and how the efforts of large majority of the population might be vane if a minority of highly active node does not change behaviour. Also, Fig. 2-d confirms the validity of the theoretical analysis. Indeed, in all scenarios considered the $\sigma_{r_{\infty}} / \sigma_{r_{\infty}}^{\max }$ estimated in the simulations are well peaked around the analytical predictions.

\section{The role of communities}

Moving forward we switch to the second, more realistic, time-varying network model. Here, the link creation mechanism is a function of nodes' membership to tightly connected groups, i.e., communities. The analysis that follows is based only on numerical simulations. Indeed, as mentioned above we do not have a closed-form expression for the threshold of activity-driven networks with communities even in the absence of behavioural change [34]. The presence of a modular structure allows us to study two different types of changes in behaviours. The first is analogous to what we presented above: as a way to protect themselves and the others, nodes reduce their social propensity. We label this as activity reduction (AR). The second instead takes inspiration from the idea of social bubbles [58]. Indeed, individuals might keep the same social propensity but direct it only towards a limited group of people. To this end, we hypothesize that nodes keep the same activity but increment the share of intra-community connections leading to an increase in the modularity of the emergent social network. We label this as 
modularity increase (MI).

\section{Activity reduction}

As done above, in this scenario we imagine that individuals change behaviours by reducing their activity by a factor $\gamma$ if susceptible and by a factor $\psi$ if infected. In Fig. 3-a we show the behaviour of the epidemic size as a function of $\lambda$. We set $\gamma=0.8, \psi=0.1$ and $p=w=0.6$. We consider a modularity $\eta=0.6$, and for simplicity we set the size of each community to be the same $(s=10)$. Thus $60 \%$ of links are created within communities and each one is made up of ten nodes. Few observations are in order. First, as seen before, random adoption is characterized by a larger threshold and a smaller final epidemic size with respect to the other case. Therefore, when the most active nodes are not adapting their behaviour the system is more fragile to the spreading of a virus. Such effects are observed both in the epidemic size as well as in the epidemic threshold (see inset). Second, we plot as vertical lines the analytical thresholds computed in absence of communities from Eq. 6 and Eq. 20. In both cases, the presence of tightly connected groups of nodes increases the threshold. This result is in line with past research showing that high values of modularity slow down the spreading of SIR (as well as SI) models [34, 42, 43, 59-61].

\section{Modularity increase}

The explicit membership to communities allows considering also another type of behavioural change where individuals keep the same activity but reduce, as a way to lower the infection risk, ties outside their close circle of friends (community). Therefore, the system moves towards isolated social bubbles. In Fig. 3-b we show the behaviour of the epidemic size as a function of $\lambda$. All the other parameters are set equal to the previous case. In particular, the default (baseline) value of modularity is $\eta=0.6$. However, the fraction of nodes implementing social distancing measures increases the modularity according to their disease status: susceptible nodes are characterized by $\eta_{\gamma}>\eta$ and infected nodes by $\eta_{\psi}>\eta$. In order to compare this scenario with the previous one, we set parameters such that the variation of behaviours has the same magnitude. More in detail, susceptible nodes change their behaviour by $20 \%$ (previously we set $\gamma$ to 0.8 , implying a $20 \%$ reduction of activity) and infected by $90 \%$. Therefore, behavioural change induce a $\eta_{\gamma}=0.68$ and $\eta_{\psi}=0.96$, which correspond to a reduction of links outside communities of $20 \%$ and $90 \%$ respectively. Also in this case, random adoption has a bigger impact on the spreading and results in a larger epidemic threshold (see inset).

By comparing the y-scales of the two scenarios presented in Fig. 3-a-b, it is clear that the reduction of activity (AR) implies a lower epidemic size with respect to the increase of

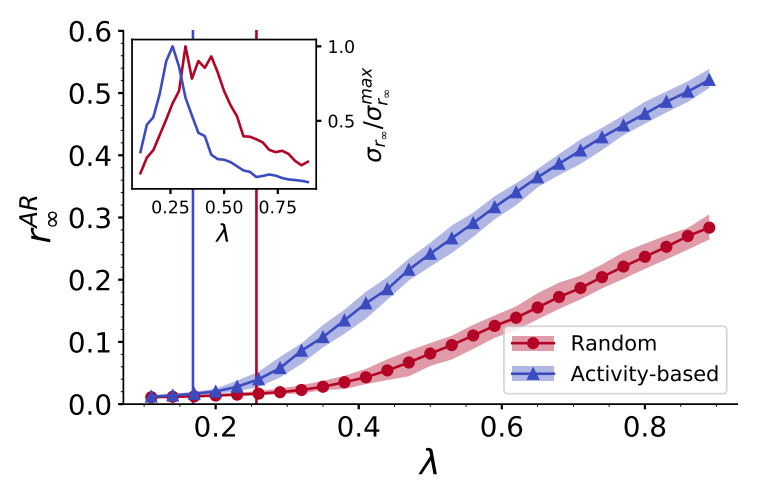

(a)

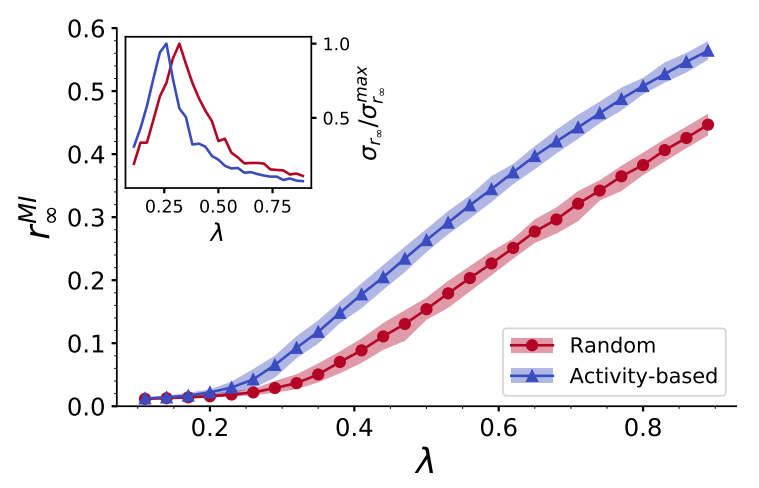

(b)

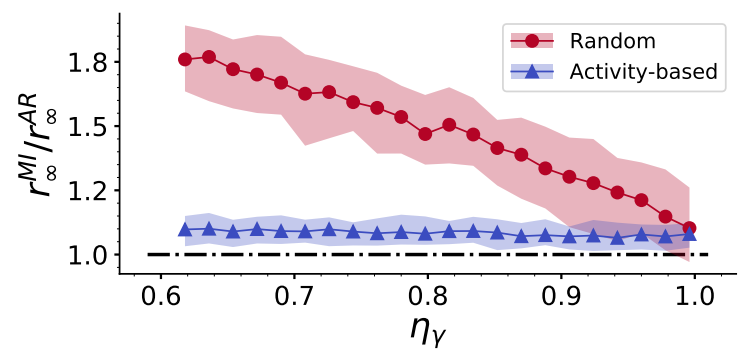

(c)

FIG. 3: a) Epidemic size as a function of $\lambda$ in presence of communities and behavioural change modeled with activity reduction. We consider both the case in which adoption is assigned randomly (red) and in increasing order of activity (blue). Vertical lines indicate the analytical threshold in absence of communities computed from Eq. 6 and Eq. 20. In the inset we represent the normalized relative variance $\sigma_{r_{\infty}} / \sigma_{r_{\infty}}^{\max }$ for different values of $\lambda$ in the case of non-perfect random and activity-based adoption in presence of communities. We set $\gamma=0.8, \psi=0.1, p=w=0.6$, $\eta=0.6, s=10$. b) Epidemic size as a function of $\lambda$ in presence of communities and behavioural change modeled with modularity increase. Also in this case we represent in the inset the normalized relative variance $\sigma_{r_{\infty}} / \sigma_{r_{\infty}}^{\max }$ for different values of $\lambda$. We set $\eta=0.6, \eta_{\gamma}=0.68, \eta_{\psi}=0.96, p=w=0.6$. c) Ratio between the final epidemic size obtained in the MI and the AR case as a function of $\eta_{\gamma}$. We set $\lambda=0.6, \eta=0.6, \eta_{\psi}=0.99, p=w=0.6$. In all figures, we represent median and $95 \%$ confidence intervals obtained from $10^{2}$ stochastic simulations for each point with the following parameters

$\mu=10^{-2}, \epsilon=10^{-3}, \alpha=2.1, N=10^{5}$ and $i_{0}=I_{0} / N=0.01$ (initial fraction of infected seeds). 
modularity (MI). We further investigate this point in Fig. 3c. To compare these two very different kinds of behavioural change, we fix all the parameters and we study the ratio of the epidemic sizes as a function of modularity of susceptible nodes. In particular, we set $\lambda=0.6, \eta=0.6, \eta_{\psi}=0.99$, $p=w=0.6$, and plot $r_{\infty}^{M I} / r_{\infty}^{A R}$ as a function of $\eta_{\gamma}$. In other words, we compare the epidemic size obtained when $60 \%$ of nodes reduce activity with what happens when they increase network modularity. The first observation is that, both in case of random and activity-based adoption, the MI strategy leads to higher epidemic sizes. Indeed, the obtained ratio $r_{\infty}^{M I} / r_{\infty}^{A R}$ is always greater than one. However, while in the case of random adoption increasing the modularity of susceptible nodes has a strong effect on the epidemic size (which progressively decreases), this effect is not observed in case of activity-based adoption. This result further confirms how the spreading patterns are largely controlled by nodes in high activity classes that do not comply with the social distancing measures.

In Fig. 4, we show the epidemic size for MI and AR as function of $\eta$. We fix $\lambda=0.6$ and study the variation of the final fraction of the population affected by the disease assuming that susceptible reduce their behaviour by $20 \%$ and infected by $90 \%$. As result, in case of modularity increase MI, the values of $\eta_{\gamma}$ and $\eta_{\psi}$ change in such a way that the probability of creating a link across bubbles (i.e., $1-\eta$ ) is reduced by $20 \%$ and $90 \%$ respectively. In panel a) we show random adoption and in panel b) adoption as function of nodes' activity. The plots confirm how MI is associated to large impact of the disease and how the differences decrease as a function of modularity $\eta$. In fact, for small values of modularity social bubbles are very loose and thus far from being effective in controlling the spreading of the virus. Behavioural changes induce a reduction of links between them but such connections are still too many and lead to a higher burden of the disease with respect to an overall reduction of activity. As noted above, the differences between the two types of behavioural changes are more evident in case of random adoption. This highlights one more time the key role of highly active individuals in driving the spreading.

\section{CONCLUSIONS}

In this work, we studied the effects of self-initiated behavioural change on disease resurgence using activity-driven networks as a modeling framework for social interactions. We imagined a population that experienced a first wave of infections that was stopped early through strict top-down interventions and did not develop significant immunity to prevent a second wave. We focused on the reactions of individuals' that, when restrictions are lifted, may adopt behavioural measures aimed at protecting themselves by reducing or changing their social interactions. This scenario is unfortunately extremely realistic. After the easing of the strict measures established during spring 2020, most Western countries faced a second wave of the COVID-19 pandemic in the winter, hence a dramatic resurgence of the virus.

More in detail, we modeled behavioural change by reducing

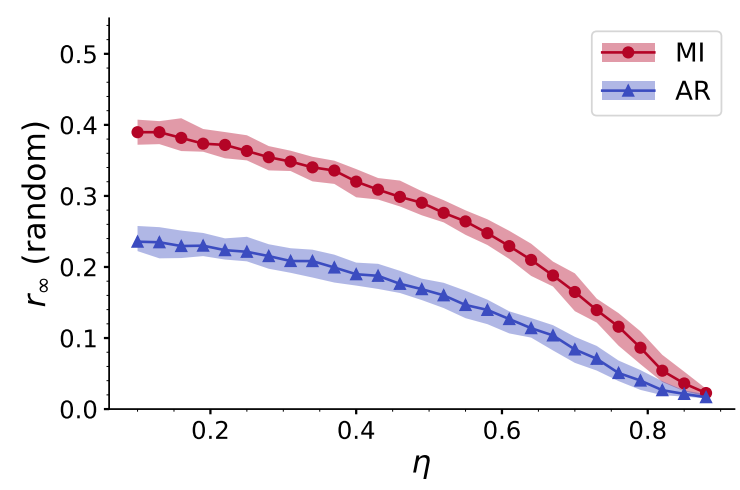

(a)

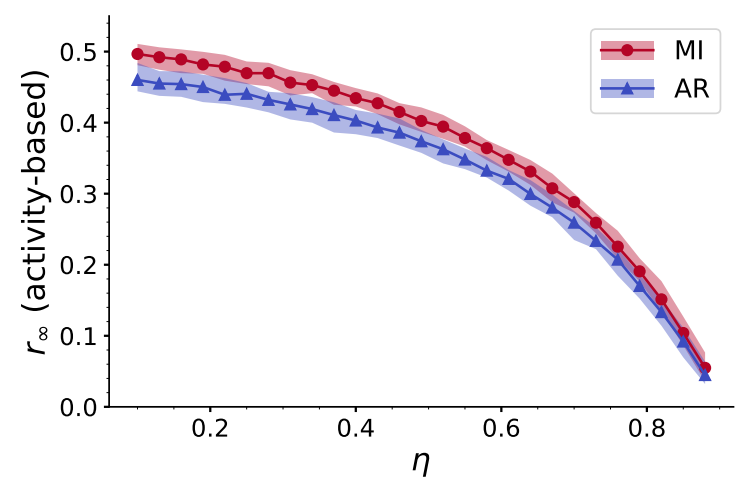

(b)

FIG. 4: Epidemic size as a function of $\eta$ in presence of communities and behavioural change modeled with activity reduction (blue) and modularity increase (red). In a) we study the case in which adoption is assigned randomly, in b) the case in which adoption is assigned in increasing order of activity. In both figures, we represent median and $95 \%$ confidence intervals obtained from

$10^{2}$ stochastic simulations for each point with the following parameters $\mu=10^{-2}, \epsilon=10^{-3}, \alpha=2.1, N=10^{5}, \lambda=0.6$, $p=w=0.6, s=10$, and $i_{0}=I_{0} / N=0.01$ (initial fraction of infected seeds). Furthermore, in both figures we assume that the susceptible reduce their behaviours by $20 \%$ and infected by $90 \%$.

the activity of susceptible and infected individuals and, having in mind the idea of social bubbles, by increasing the share of connections within tight social circles with respect to their baseline. In doing so, we explored the effect of behaviour adoption by considering only a fraction of nodes engaging in behavioural protective measures selected either at random or as function of their activity. In fact, the most socially active nodes, maybe due to the nature of their job, cannot easily modify their behaviours.

Considering first the simplest version of activity-driven networks, where links are memory-less and random, we derived the analytical threshold of a Susceptible-Infected-Recovered epidemic model. In doing so, we extended the work done by Rizzo et al [21] accounting for non-perfect adoption. Interestingly, in case nodes adapting their behaviours are selected randomly in the population, we found that the expression for the basic reproductive number $R_{0}$ is symmetrical with respect a combination of activity reduction and level of adoption of 
susceptible and infected. Given the high socio-economic cost associated with isolating large numbers of people, this finding underlines the importance of efficient test and trace systems to isolate infected, especially at the beginning of a possible second wave when their number is relatively small. Furthermore, the numerical simulations showed that, in the settings considered, the final epidemic size was mainly dependent on the reduction in activity of the infected. In case adherence is assigned in increasing order of activity, we found that an almost perfect level of adoption is needed in order to avoid the disease to rise again. This effect is deeply connected to the high heterogeneity of human interactions, and it highlights that even small levels of lack of adoption by very active nodes may have a huge impact on the spreading. This finding is in line with previous studies of targeted immunization strategies on time-varying networks which show how immunizing the most highly active nodes is extremely effective in hampering the spreading of contagion phenomena [22, 28, 46, 62]. Interestingly, the critical role of a small number of individuals and locations (i.e., super-spreaders) has been reported and observed in several publications focused on the COVID-19 pandemic [63-65]. Intense social contacts, crowds, as well as environmental factors such as indoor settings and poor ventilation have been identified as important factors driving such events.

We then moved to even more realistic scenarios, taking into account the tendency of people to cluster in tightly connected groups. To this aim, we considered a modified formulation of the activity-driven model in which nodes are assigned to communities and tend to establish links inside their community more often than with outside nodes [34]. In this setting, we modeled behavioural change by considering two mechanisms: i) reducing the activity of nodes and ii) keeping the same level of activity but limiting the contacts outside communities and thus increase network's modularity. Using numerical simulations, we observed that the modularity of the network increases the threshold with respect to the previous case. This is in line with past observations on synthetic and real timevarying networks $[34,42,43,47,59,60,66]$. Furthermore, random adoption is characterized by a larger threshold and a smaller epidemic size with respect to the case of adoption assigned in increasing order of activity. Finally, we found that an activity reduction strategy is more efficient than increasing the modularity across the range of parameters studied. This finding highlights how imperfect social bubbles might not be as effective as an overall reduction of social activities. In real world scenarios the impact of social bubbles is affected by a range of variables and implementation details. Indeed, a recent study conducted by calibrating an individual based epidemic model to the COVID-19 pandemic in Belgium, shows how strictness levels (i.e., which map to our concept of modularity), intergenerational mixing in households, and the implementation of complementary measures such as contact tracing among others factors affect their impact [67].

Of course, this work comes with limitations. First of all, our contribution is only theoretical and limited by the set of assumptions that were made. As such, it should not be indented as a precise representation of reality and especially of the current pandemic landscape. Although we focused on some fundamental features of realistic epidemiological models (e.g., heterogeneity of contacts, modularity, behavioural change), we overlooked many others such as including several connected populations, considering an age-structured population, the complex nature of real self-initiated behavioural change, or transmission dynamics which might include presymptomatic or asymptomatic carries [1, 2, 7, 8, 65, 68, 69]. We have limited ourselves to an exploration of the phase space rather than fitting the parameters using real data. We have neglected high-order complex temporal dynamics of real timevarying networks such as real face-to-face networks [70-73], and non-Markovian features of both connectivity patterns and epidemic processes [30,52-54]. We leave these extensions and model calibration for the future.

In conclusion, our work contributes to the characterization of self-initiated behavioural change in the context of disease resurgence on time-varying networks. It highlights the importance of accounting for the heterogeneity of social activation patterns when gauging the efficiency of adaptive strategies aimed at hampering the spreading of infectious diseases on temporal networks.

\section{ACKNOWLEDGEMENTS}

All authors thank the High Performance Computing facilities at Greenwich University. N.G. acknowledges support from the Doctoral Training Alliance. N.P. thanks Dr. Michele Starnini for useful conversations.
[1] S. Funk, M. Salathé, and V. A. A. Jansen, Journal of The Royal Society Interface 7, 1247 (2010), https://royalsocietypublishing.org/doi/pdf/10.1098/rsif.2010.0142.

[2] F. Verelst, L. Willem, and P. Beutels, Journal of The Royal Society Interface 13 (2016), 10.1098/rsif.2016.0820, https://royalsocietypublishing.org/doi/ pdf/10.1098/rsif.2016.0820.

[3] S. Funk, S. Bansal, C. T. Bauch, K. T. Eames, W. J. Edmunds, A. P. Galvani, and P. Klepac, Epidemics 10, 21 (2015), challenges in Modelling Infectious DIsease Dynamics.
[4] T. Gross and B. Blasius, Journal of the Royal Society Interface 5, 259 (2008).

[5] V. Marceau, P.-A. Noël, L. Hébert-Dufresne, A. Allard, and L. J. Dubé, Physical Review E 82, 036116 (2010).

[6] N. Perra, Physics Reports https://doi.org/10.1016/j.physrep.2021.02.001.

[7] I. M. Rosenstock, Health Education Monographs 2, 354 (1974), https://doi.org/10.1177/109019817400200405.

[8] G. Hochbaum, Public Participation in Medical Screening Programs: A Socio-psychological Study, Public Health Service 
publication (U.S. Department of Health, Education, and Welfare, Public Health Service, Bureau of State Services, Division of Special Health Services, Tuberculosis Program, 1958).

[9] R. West, S. Michie, G. J. Rubin, and R. Amlôt, Nature Human Behaviour, 1 (2020).

[10] N. Gozzi, D. Perrotta, D. Paolotti, and N. Perra, PLoS computational biology 16, e1007879 (2020).

[11] G. Guzzetta, F. Riccardo, V. Marziano, P. Poletti, F. Trentini, A. Bella, X. Andrianou, M. Del Manso, M. Fabiani, S. Bellino, et al., arXiv preprint arXiv:2004.12338 (2020).

[12] N. Perra, D. Balcan, B. Gonçalves, and A. Vespignani, PloS one 6 (2011).

[13] A. Moinet, R. Pastor-Satorras, and A. Barrat, Physical Review E 97, 012313 (2018).

[14] S. Meloni, N. Perra, A. Arenas, S. Gómez, Y. Moreno, and A. Vespignani, Scientific reports 1, 62 (2011).

[15] M. Chinazzi, J. T. Davis, M. Ajelli, C. Gioannini, M. Litvinova, S. Merler, A. P. y Piontti, K. Mu, L. Rossi, K. Sun, et al., Science (2020).

[16] M. U. Kraemer, C.-H. Yang, B. Gutierrez, C.-H. Wu, B. Klein, D. M. Pigott, L. du Plessis, N. R. Faria, R. Li, W. P. Hanage, et al., Science 368, 493 (2020).

[17] L. Hébert-Dufresne, D. Mistry, and B. M. Althouse, arXiv preprint arXiv:2003.10604 (2020).

[18] C. Granell, S. Gómez, and A. Arenas, Physical review letters 111, 128701 (2013).

[19] M. Mancastroppa, R. Burioni, V. Colizza, and A. Vezzani, arXiv preprint arXiv:2004.07902 (2020).

[20] T. Gross, C. J. D. D'Lima, and B. Blasius, Physical review letters 96, 208701 (2006).

[21] A. Rizzo, M. Frasca, and M. Porfiri, Physical Review E 90, 042801 (2014).

[22] Z. Wang, C. T. Bauch, S. Bhattacharyya, A. d'Onofrio, P. Manfredi, M. Perc, N. Perra, M. Salathé, and D. Zhao, Physics Reports 664, 1 (2016).

[23] S. Herzog, J. De Bie, S. Abrams, I. Wouters, E. Ekinci, L. Patteet, A. Coppens, S. De Spiegeleer, P. Beutels, P. Van Damme, N. Hens, and H. Theeten, medRxiv (2020), 10.1101/2020.06.08.20125179, https://www.medrxiv.org/content/early/ 2020/06/09/2020.06.08.20125179.full.pdf.

[24] S. Stringhini, A. Wisniak, G. Piumatti, A. S. Azman, S. A. Lauer, H. Baysson, D. De Ridder, D. Petrovic, S. Schrempft, K. Marcus, I. Arm-Vernez, S. Yerly, O. Keiser, S. Hurst, K. Posfay-Barbe, D. Trono, D. Pittet, L. Getaz, F. Chappuis, I. Eckerle, N. Vuilleumier, B. Meyer, A. Flahault, L. Kaiser, and I. Guessous, medRxiv (2020), 10.1101/2020.05.02.20088898, https://www.medrxiv.org/content/early/ 2020/05/06/2020.05.02.20088898.full.pdf.

[25] M. O'Driscoll, G. R. Dos Santos, L. Wang, D. A. Cummings, A. S. Azman, J. Paireau, A. Fontanet, S. Cauchemez, and H. Salje, Nature (2020).

[26] P. Holme and J. Saramäki, Physics reports 519, 97 (2012).

[27] P. Holme, The European Physical Journal B 88, 234 (2015).

[28] N. Masuda and P. Holme, Temporal network epidemiology (Springer, 2017).

[29] N. Perra, B. Gonçalves, R. Pastor-Satorras, and A. Vespignani, Scientific reports 2, 469 (2012).

[30] M. Karsai, N. Perra, and A. Vespignani, Scientific reports 4, 4001 (2014).

[31] E. Ubaldi, N. Perra, M. Karsai, A. Vezzani, R. Burioni, and A. Vespignani, Scientific reports 6, 35724 (2016).
[32] E. Ubaldi, A. Vezzani, M. Karsai, N. Perra, and R. Burioni, Scientific reports 7, 46225 (2017).

[33] L. Alessandretti, K. Sun, A. Baronchelli, and N. Perra, Physical Review E 95, 052318 (2017).

[34] M. Nadini, K. Sun, E. Ubaldi, M. Starnini, A. Rizzo, and N. Perra, Scientific reports 8, 1 (2018).

[35] B. Ribeiro, N. Perra, and A. Baronchelli, Scientific reports 3, 3006 (2013).

[36] M. V. Tomasello, N. Perra, C. J. Tessone, M. Karsai, and F. Schweitzer, Scientific reports 4, 5679 (2014).

[37] M. J. Keeling and P. Rohani, Modeling infectious diseases in humans and animals (Princeton University Press, 2011).

[38] S. Fortunato, Physics reports 486, 75 (2010).

[39] A. Moinet, M. Starnini, and R. Pastor-Satorras, Physical review letters 114, 108701 (2015).

[40] T. Brett, G. Loukas, Y. Moreno, and N. Perra, Physical Review E 99, 050303 (2019).

[41] M. Starnini and R. Pastor-Satorras, Physical Review E 87, 062807 (2013).

[42] J.-P. Onnela, J. Saramäki, J. Hyvönen, G. Szabó, D. Lazer, K. Kaski, J. Kertész, and A.-L. Barabási, Proceedings of the national academy of sciences 104, 7332 (2007).

[43] M. Karsai, M. Kivelä, R. K. Pan, K. Kaski, J. Kertész, A.L. Barabási, and J. Saramäki, Physical Review E 83, 025102 (2011).

[44] G. Miritello, E. Moro, and R. Lara, Physical Review E 83, 045102 (2011).

[45] N. Perra, A. Baronchelli, D. Mocanu, B. Gonçalves, R. PastorSatorras, and A. Vespignani, Physical review letters 109 , 238701 (2012).

[46] S. Liu, N. Perra, M. Karsai, and A. Vespignani, Phys. Rev. Lett. 112, 118702 (2014).

[47] J. T. Davis, N. Perra, Q. Zhang, Y. Moreno, and A. Vespignani, Nature Physics 16, 590 (2020).

[48] M. Starnini and R. Pastor-Satorras, Physical Review E 89, 032807 (2014).

[49] L. Zino, A. Rizzo, and M. Porfiri, Physical review letters 117, 228302 (2016).

[50] L. Zino, A. Rizzo, and M. Porfiri, Journal of Complex Networks 5, 924 (2017).

[51] R. Pastor-Satorras, C. Castellano, P. Van Mieghem, and A. Vespignani, Reviews of modern physics 87, 925 (2015).

[52] M. Boguná, L. F. Lafuerza, R. Toral, and M. Á. Serrano, Physical Review E 90, 042108 (2014).

[53] P. Van Mieghem and R. Van de Bovenkamp, Physical review letters 110, 108701 (2013).

[54] L. Böttcher and N. Antulov-Fantulin, Phys. Rev. Research 2, 033121 (2020).

[55] M. Starnini, J. P. Gleeson, and M. Boguñá, Physical review letters 118, 128301 (2017).

[56] K. Sun, A. Baronchelli, and N. Perra, The European Physical Journal B 88, 1 (2015).

[57] M. Tizzani, S. Lenti, E. Ubaldi, A. Vezzani, C. Castellano, and R. Burioni, Physical Review E 98, 062315 (2018).

[58] T. Leng, C. Whie, J. Hilton, A. J. Kucharski, L. J. Pellis, H. Stage, N. G. Davies, M. J. Keeling, and S. Flasche, medRxiv (2020).

[59] M. Salathé and J. H. Jones, PLoS Comput Biol 6, e1000736 (2010).

[60] C. Stegehuis, R. Van Der Hofstad, and J. S. Van Leeuwaarden, Scientific reports 6, 1 (2016).

[61] S. V. Scarpino, A. Allard, and L. Hébert-Dufresne, Nature Physics 12, 1042 (2016). 
[62] C. L. Vestergaard, M. Génois, and A. Barrat, Physical Review E 90, 042805 (2014).

[63] M. P. Kain, M. L. Childs, A. D. Becker, and E. A. Mordecai, Epidemics , 100430 (2020).

[64] M. S. Lau, B. Grenfell, M. Thomas, M. Bryan, K. Nelson, and B. Lopman, Proceedings of the National Academy of Sciences 117, 22430 (2020).

[65] E. C. Lee, N. I. Wada, M. K. Grabowski, E. S. Gurley, and J. Lessler, Science 370, 406 (2020).

[66] D. Han, M. Sun, and D. Li, Physica A: Statistical Mechanics and its Applications 432, 354 (2015).

[67] L. Willem, S. Abrams, P. J. Libin, P. Coletti, E. Kuylen, O. Petrof, S. Møgelmose, J. Wambua, S. A. Herzog, C. Faes, et al., Nature communications 12, 1 (2021).

[68] J. Hayden, Introduction to Health Behavior Theory (Jones \& Bartlett Learning, 2013).

[69] G. St-Onge, V. Thibeault, A. Allard, L. J. Dubé, and L. HébertDufresne, arXiv preprint arXiv:2003.05924 (2020).

[70] R. Lambiotte, M. Rosvall, and I. Scholtes, Nature physics 15, 313 (2019).

[71] F. Battiston, G. Cencetti, I. Iacopini, V. Latora, M. Lucas, A. Patania, J.-G. Young, and G. Petri, Physics Reports (2020).

[72] G. Petri and A. Barrat, Physical review letters 121, 228301 (2018).

[73] C. Cattuto, W. Van den Broeck, A. Barrat, V. Colizza, J.-F. Pinton, and A. Vespignani, PloS one 5, e11596 (2010). 